\title{
GAMIFICATION IN KNOWLEDGE MANAGEMENT SYSTEMS
}

\author{
Michal Ďuriník
}

\begin{abstract}
While computer games are extremely immersing and engaging, many companies find their knowledge management systems (KMS) to be ignored by the employees. Gamification is a way of enhancing KMS with game design elements to increase user engagement, content creation and satisfaction. This paper discusses what gamification in KMS is, what the game design elements to be used are, and how companies can benefit from implementing gamified KMS. Furthermore, this paper provides an overview of psychological mechanisms that drive user behavior and attitudes when using gamified KMS.
\end{abstract}

\section{Key words}

Gamification, knowledge management systems, KMS, knowledge management.

\section{Introduction}

Many companies have developed and launched new knowledge management systems (KMS) only to watch them be ignored by the employees. These employees, on the other hand, are the same people who spend hours of their free time chasing ogres and crushing candies in addictive online videogames. This paper discusses KMS gamification - the use of game elements in KMS design in order to increase employee engagement. The paper aspires to be a general introduction to the topic, providing readers with definition and more in-depth look at various gamification elements. The paper also outlines psychological mechanisms driving user behavior and attitudes in gamified KMS, and benefits and controversies associated with gamification are briefly discussed.

In today's knowledge economy, the experiences, ideas, insights and intellectual capital possessed by the employees is one of the greatest company assets. Some authors (Liebeskind, 1996, for instance) even regard it as the most valuable resource in a company. Companies learn to harness the pieces of knowledge scattered among the employees to gain the ability to respond to a wide range of challenges (Hansen \& von Oetinger, 2001). Unlike some other company assets, knowledge provides the most value when it is shared - sharing knowledge enhances decision making, improves performance and facilitates the development of new knowledge (Brown, Dennis, Burley, \& Arling, 2013). To aid knowledge sharing and use, companies have started to develop and launch knowledge management systems. In many cases however, they found that employees do not use them (Hansen \& von Oetinger, 2001). Employees either did not share knowledge at all, or they avoided using KMS and shared knowledge on a person-toperson basis (Wasko \& Faraj, 2005).

Any KMS, even a well-designed one, is worthless if it is not used. Brown, Dennis, Burley and Arling (2013) provide a summary of key problems, that influence KMS usage by employees: motivation to share knowledge, establishing trust, cultivating positive attitudes towards 
knowledge sharing and KMS, creating effective processes for sharing knowledge and assessing KMS success. There are several ways to increase employees' motivation, improve their attitude and help them find meaning and joy in sharing the knowledge they possess. Videogame designers have pioneered the use of points, badges, challenges and leaderboards to create games that are attractive, and even addictive (Barnes \& Pressey, 2014; Heaven, 2014). These game elements can be also adopted for the workplace, school, website or software application to improve performance and user experience by what is called gamification (Pedreira, García, Brisaboa, \& Piattini, 2014).

Gamification is being used in many areas these days (Hamari, Koivisto, \& Sarsa, 2014), often to help users learn new skills or adopt a habit. Some examples you can use with your own smartphone are Duolingo (to learn a language), Plant Nanny (to learn to drink more water through the day) and Fitocracy (to increase physical fitness) - all of these use points, progress, levels, immediate feedback and other game elements to influence users' behavior.

\section{Gamification}

Deterding, Dixon, Khaled and Nacke (2011) provide the following definition: Gamification is the use of game design elements in non-game contexts. We shall take a look at particular components of this definition in the following section. First, we need to define what a game is in this context.

As Deterding et al. (2011) note, gamification refers to games, not play. Play can be described as expressive, freeform and improvisational activities and combinations of behaviors, while gaming is a more structured version of play with rules and competitiveness. In contrast to playing, games have rule systems one needs to follow and participants in games are oriented towards reaching certain goals or outcomes (Deterding et al., 2011). In encouraging employees to engage in KMS usage more, it is desirable to lean more towards game than play - users purposelessly interacting with and wandering through a KMS environment may be having fun, but this activity provides little to no value. Having pre-set goals, rule sets and reward mechanisms (more on these later) helps users stay motivated and focused on interacting with KMS content in a meaningful way.

Discussing what is a game element, Deterding et al. (2011) admit that the boundaries here are blurred and there is much room for discussion over what game elements really are. There are two extreme poles that would render either an empty set of elements found in all games, or a nearly infinite set of elements found at least in some games. Deterding et al. (2011) propose a definition in between these two poles, considering elements that are characteristic to games: game elements are elements found in most (but not necessarily all) games, readily associated with games, and found to play a significant role in gameplay. A list of game element examples can be found in Reeves \& Read (2009): Self-representation with avatars, 3D environments, narrative context, feedback, reputations, ranks and levels, marketplaces, competition under explicit and enforced rules, teams and time pressure. Some of these elements can be effectively used in gamifying KMS (feedback, ranks, ...) while others, such as 3D environments, could be an unnecessary distraction.

Speaking of non-game contexts, Deterding et al. (2011) refer to uses of game elements for other purposes than in entertainment games. These purposes may be to motivate, to educate, to build habits or to provide training. To fulfill these goals, not only gamified applications may be used, but serious games also. What is the difference between gamified applications 
and serious games? The former is a regular application (promotional, educational or KMS for instance) enhanced with multiple game elements to improve user experience and engagement. A serious game, on the other hand, has all the requirements of being a game - it is a full game with all the game aspects, even though it fulfills non-entertainment goals while being fun (DeSmet et al., 2014). A serious game is intended to be played in its entirety from start to end to provide the non-entertaining benefit (Rinc, 2014). Gamified applications can be engaged with (utilized?) in bits and pieces over time without any harm to the experience - the employee simply interacts with the KMS in a meaningful way and is rewarded with a certain amount of points or receives a new badge.

\section{Game elements in KMS gamification}

Companies can use multiple game elements in gamifying knowledge management systems. This chapter discusses several of these elements in greater detail. Some elements construct the game mechanics (points and badges that provide feedback on participant's progress), while others build up the replication mechanics (leaderboards that make participant's performance level public) and social mechanics (peer ratings and comments that help to build relationships within the gamified application) (Wolfinger, 2013).

Game elements that can be used to gamify KMS include (Trees, 2013):

- Points

- Badges

- Challenges and Contests

- Awards and Prizes

- Rankings and Leaderboards

The fundamental part of any gamified system is the ability for users to earn points. Users are rewarded with points for performing various tasks, such as contributing to the shared knowledge base, submitting a wiki article, replying to a discussion forum question or posting to a blog (Hamari, 2013; Trees, 2013). There is no monetary value to the points, as they cannot be exchanged for cash or a pay raise. They can, however, "buy" badges, ranks, leaderboard placement and in some cases even some tangible rewards (see below). Moreover, users are motivated by social pressure and competition to accumulate more points than other users, and may also be motivated by the mere satisfaction of having more points than before. When designing gamified KMS, it is important to set up the point-awarding mechanism transparently, so the users know what activities are rewarded with what amounts of points.

Badges are ornaments or symbols users can earn for pre-defined favorable behaviors (Hamari, 2013). These behaviors may include reaching thresholds in point accrual, completing certain tasks, winning challenges, performing certain task for several consecutive days and so on. When the user earns a badge, he can display it in his company profile or attach it to his avatar for everyone to see (Deterding et al., 2011). Public display of badges allows for comparison with others, delivering satisfaction to those with many hard-to-earn badges, and motivating those who are below the average. Just like in children's scouting, badges are awarded for reaching a pre-set goal. In gamified KMS however, badges are virtual only and are awarded for reaching different goals. For instance, user can be rewarded with "Emerging Blogger" badge for submitting 3 blog posts or with "VAT Ninja" for answering 100 VAT-related questions. Badges represent goals and according to Bandura (1993), goal-setting increases 
performance in three ways: 1) with goals, people anchor their expectations higher, this in turn increases their performance; 2) self-efficacy is enhanced with assigned goals; and 3) upon goal completion users' satisfaction increases, leading to increased performance in the same activity in the future. Badges themselves are a valid motivational tool and mere proximity of reaching a badge threshold may result in a peak in performance just to receive the badge earlier. To foster this, reminders such as "only 2 more blog posts to your Advanced Blogger badge!" or "write 10 more valuable feedback comments to earn your Feedback Star badge!" are useful and easy to implement in the gamified KMS.

Challenges and Contests can also be included in gamified KMS. These tasks are typically limited in time: users have a pre-set timeframe to fulfill them - two hours, a day or a week (Trees, 2013). Challenges are tasks that need to be fulfilled in the time given in order to receive a reward - a badge or a pre-defined amount of points. In contests, users compete for the best performance within a given timeframe. Challenges and contests put users under time pressure, which according to Andrews \& Farris (1972) increases several aspects of performance including usefulness, innovation and productivity. Turning a task into a time-constrained challenge renders it to be of higher priority. Users who decide to pursue the challenge allocate more resources to task-relevant activities and avoid distractions more (Baumann, 1998). Setting up challenges is not optimal for all kinds of tasks though. Time pressure increases performance, but at the same diminishes precision and output quality (Moore \& Tenney, 2012). Users under time pressure rely more heavily on various heuristics and their focus scope is narrowed. Thus time-limited challenges and contests are not suitable for creative tasks or those that require great precision and analytical approach. On the other hand, they may prove beneficial for simple and repetitive tasks, such as "In the next hour, share your latest KMS contribution with 5 of your colleagues who might benefit from it".

Rankings and Leaderboards represent the cumulative results of points collected, challenges won and badges earned (Trees, 2013). These are well known in the world of video games and provide additional motivation for extended effort and engagement. Being the best, or among the best in a certain area and having others see my success in the leaderboard motivates me to earn more points and badges. Thus, rankings and leaderboards attenuate the peer pressure and competition factor of gamification even more.

Achieving a certain ranking, earning a certain badge or accumulating given number of points may even have some real-world outcomes. Some gamification strategies include rewards that exist outside the gamified KMS environment. These may include tangible rewards such as gift cards, or intangible and career-oriented rewards such as an attractive business trip, face time with company leaders or the opportunity to attend the board meeting (Trees, 2013; Wang \& Lai, 2014). Whether monetary rewards result in desired results or not is not clear, however. Wang \& Lai (2014) found a system of tangible rewards to improve KMS adoption and usage, while Bock, Ko, Kirsch, \& King (2005) found that the expectation of monetary reward for knowledge sharing was negatively related to attitudes toward knowledge sharing.

\section{The Psychology of Gamification}

Tangible rewards of using gamified KMS are rare and in many cases non-existent, therefore we cannot rely on monetary incentives as an explanation for employees' engagement. In psychological literature, however, we can identify several concepts that can be used to (partially) explain the inner workings of gamification. 
We start with the very cornerstone of motivation theory: Abraham Maslow's hierarchy of needs, as it was presented in his 1943 paper. Maslow divided needs into several groups, in order from the most basic: physiological; safety; love and belonging; esteem; and self-actualization needs. Unlike earning cash, earning points and badges cannot satisfy the Safety needs, for it can fulfill neither the security of resources or property, nor the security of employment. Using gamified KMS can however satisfy the "higher" needs, especially in the Esteem level of the pyramid. As Maslow puts it, people have the need for high evaluation of themselves, for self-esteem, self-respect and the esteem of others. Earning badges and points and being among the best performers on the leaderboard is confirmation that one has provided valuable content and is recognized and acknowledged for it. This in turn, in Maslow's (1943) words "[...] leads to feelings of self-confidence, worth, strength, capability and adequacy of being useful and necessary in the world." Feeling strong, worthy and capable, in turn increases the effort and engagement one produces (Meder, Plumbaum, Luca, \& Albayrak, 2011). We can say that gamification allows KMS users to experience satisfaction of needs that would be difficult to satisfy without it.

An important aspect of gamified application is the immediate feedback that points, challenges and badges provide. Instead of calculating the points at the end of the month for example, it is more beneficial to award them immediately upon completion of a certain task. This way immediate feedback is provided, the behavior-reward delay is minimized and the user is more likely (compared to the delayed scenario) to engage in KMS activity again. Immediate feedback with a sense of control, progress tracking and the ability to succeed in challenges are qualities of gamified KMS experience, but also they are the qualities of the experience of Flow (Csikszentmihalyi, Abuhamdeh, \& Nakamura, 2005). Flow, as defined by Csikszentmihalyi (1990), is a state of optimal experience characterized as being fully focused and engaged in an activity, even to the extent of losing track of time while immersed in the activity. In their recent paper, Hamari and Koivisto (2014) validate the Dispositional Flow Scale-2 in the context of gamification providing additional evidence for close link between the flow experience and the gamified experience.

One's own judgments about his or her capabilities of executing the courses of action required to achieve specific goal or performance are referred to as Self-efficacy (Bandura, 2012). As self-efficacy is related to the amount of effort and persistence one exerts towards a focal behavior (Hasan, 2006), it is argued that it plays a vital role in influencing one's motivation and behavior regarding the use of IT (Ambrose \& Chiravuri, 2010), including knowledge management systems. KMS self-efficacy relates to more than just a user's computer skills, it reflects his ability to use KMS to accomplish specific tasks (Lai, 2009). Wang and Lai (2014) find KMS self-efficacy to be of importance in relation to KMS adoption and use, but identify other factors (top management support, organizational rewards and system quality factors) to be more effective in shaping KMS-related behaviors and beliefs in employees. If gamified KMS can provide more immediate feedback and a better sense of accomplishment and recognition, it also increases user's KMS self-efficacy. Employees with greater KMS self-efficacy, in turn, tend to have greater willingness and capabilities to engage with KMS more often and contribute more valuable content (Kuo \& Lee, 2011).

As noted earlier, games and gamified systems incorporate competition between users in one way or the other. In gamified KMS too, collecting points, badges and rewards can be a source of competitive behaviors and emotions. Contributing to KMS more than others can 
be a source of prestige (He \& Wei, 2009), nudging those who seek and enjoy prestige towards greater engagement in KMS. Competition in collecting points cannot exist without the sense of peer pressure and rivalry, which too, increases one's motivation and performance (Kilduff, 2014).

Not all people are the same when it comes to motivation, performance, adoption and usage of IT or attitudes towards knowledge sharing - there are individual differences in all these areas. Differences in KMS usage were observed between individuals of different ages, sex and various levels of psychological trait scores. Koivisto and Hamari (2014) found women reporting greater social benefits from the use of gamified application than men. This is not surprising, since in massive multiplayer online games (not gamified applications) women are motivated by immersion and social factors, while men are more achievement-oriented and display more competitiveness and need for winning than women (Hartmann \& Klimmt, 2006; Williams, Consalvo, Caplan, \& Yee, 2009).

Older users are considered to rely more on social influence in their technology adoption than young ones (Venkatesh, Morris, Davis, \& Davis, 2003). Younger employees tend to be more autonomous in their usage and adoption of IT (Morris, Venkatesh, \& Ackerman, 2005), largely because they have been exposed to digital technologies at a much younger age than older generations (Morris \& Venkatesh, 2000). As with age, learning to use a new service or device becomes more time-consuming and older users pay greater attention to ease of use as one of the key factors that facilitates the adoption of technology (Arning \& Ziefle, 2007). While supporting social environment and ease of application are important considerations with every gamified KMS launch, they become even more crucial when the employees are of a higher age.

In their quasi-field experiment, Wang, Noe, and Wang (2011) investigated how personality traits influence knowledge sharing in KMS (in this case non-gamified, although incentivized). In particular, personality traits were represented by the elements of the five-factor model of personality (McCrae \& Costa, 1989): openness to experience, conscientiousness, extroversion, agreeableness, neuroticism. Of these elements, openness to experience (individuals high in openness to experience are curious, creative and broadminded), conscientiousness (conscientious individuals are self-disciplined, dutiful, responsible and behave in a manner that meets other's expectations) and neuroticism (individuals high in neuroticism are low in emotional stability) were found to be of significant relationship with knowledge sharing in the KMS. Compared to those low in neuroticism, those high in neuroticism displayed larger increase in knowledge sharing when accountability and incentives (elements that gamification helps to elicit) were introduced into the KMS. Extending from this, we might expect employees scoring high in neuroticism to show larger increase in knowledge sharing upon introduction of gamified KMS. Similarly, employees low in conscientiousness displayed greater increase in knowledge sharing than those low in conscientiousness when accountability and incentives were introduced. Authors explain this by the fact that conscientious individuals are dutiful by nature and are less influenced by external incentives (Wang, Noe, and Wang, 2011). Low openness to experience was associated with greater increase in knowledge sharing upon the introduction of accountability and reward. Wang, Noe, and Wang (2011) explain: individuals high in openness to experience seek out knowledge more because of their natural curiosity, but do not necessarily feel the need to share it with others. 


\section{Implementing gamification in KMS}

Just as there are differences between individual people, there are differences between companies. Company culture, and at the higher level national culture too, can make the introduction of gamified KMS easy and smooth, or make it a difficult struggle. Companies differ in three levels that define corporate culture - the different cultural artifacts, espoused beliefs and values and also basic underlying assumptions (Schein, 2010). In some cases, introduction of gamified KMS can be in disharmony with the present company culture, or even in conflict with it. In this case, there is a possibility of cultural change in the company - it must be executed with care and good preparation though.

Gamification in knowledge management systems is an emerging academic field that has started to flourish in three main geographical areas: USA, (Northern) Europe and Asia (especially China). When considering the empirical results for practical application, we need to bear in mind how great the intercultural differences, as explained by Hofstede and Hofstede (2006), are. Europe and USA are highly individualistic cultures (of the top 30 countries by individualism, 26 are in North America or in Europe (Hofstede \& Hofstede, 2006)): this makes us expect that gamification measures that foster competition and rivalry (such as leaderboards and points) can have a great impact on one's engagement with the KMS. In Asia and other countries low in individualism, the competitive aspect of gamified KMS may not be such a strong motivator.

Gamified KMS is not only competition, though: points and achievements earned are recognition of one's abilities and skills and help nourish one's self-esteem (as discussed above). Therefore, gamification can be of great benefits also in cultures (both corporate and national) that do not rely on competition and individualism so heavily.

Depending on the current state of systematization of knowledge management, introducing gamified KMS may take two forms: in case there is no KMS currently used in the company, launching gamified KMS equals launching a brand new system to store, process and deliver knowledge across a company. Should there be an existing KMS, it may be enough for a company to simply enhance it with game elements. In both cases, however, it is important to introduce and explain to the employees that a new platform is being launched, what the expectations are and what it can do for the company and employees themselves.

\section{Summary}

Why should companies be interested in gamification of knowledge management systems? This paper shows evidence that gamified KMS, through points, badges, challenges, leaderboards and other game elements provides employees with the platform to compete in, enhances their self-esteem and helps to satisfy certain higher needs from Maslow's pyramid. In the end, it helps to fulfill the main goal, which is to increase employees' engagement with the KMS when it comes to contributing valuable content, organizing and improving it, or using it in a meaningful way. All these could be achieved also with a monetary reward system, but the advantage of gamification is the relatively low costs: although points and badges have to be earned by the employees, the company pays nothing to issue them. The costs of gamified KMS are therefore not much higher than the costs of non-gamified KMS (which provides less immersion, competition and satisfaction than a gamified one). 
As noted by Trees (2013), gamification is not a silver bullet to solve all KMS issues in a company. It is only a tool that can, but does not necessarily have to, help accomplish company's KMS goals. There are some disappointments that implementation of gamified KMS can bring. First, there is the possibility that, just like the original KMS, the gamified one will also be ignored and bypassed by the employees. This could arise from cultural issues, employees' individual traits, job nature (KMS is not an optimal solution for all jobs and positions) or management's failure to explain and introduce the purpose and meaning behind gamified KMS. A company may also arrive to disappointment after finding out that employees try to "game" the system: instead of interacting with KMS in meaningful ways, they perform activities that do not follow the main goals of knowledge management, simply to collect as many points as they can. In this case, it may be useful to re-explain the meaning of KMS, and also readjust the feedback and point-generating mechanisms within KMS.

This paper explained what gamification is, what elements it uses and how it can help companies fulfill their knowledge-management oriented goals. Closer attention was paid to various psychological mechanisms and their role in gamification effectiveness. Additionally, demographic and cultural factors that can be of influence in gamified KMS adoption and use were discussed.

\section{Acknowledgement}

This paper was elaborated within the framework of Masaryk University specific research project MUNI/A/0814/2013.

\section{References}

Ambrose, P. J., \& Chiravuri, A. (2010). A socio-cognitive interpretation of the potential effects of downsizing on software quality performance. Information Systems Journal, 20, 239-265. doi:10.1111/ j.1365-2575.2009.00332.x

Andrews, F. M., \& Farris, G. F. (1972). Time pressure and performance of scientists and engineers: A five-year panel study. Organizational Behavior and Human Performance. doi:10.1016/00305073(72)90045-1

Arning, K., \& Ziefle, M. (2007). Understanding age differences in PDA acceptance and performance. Computers in Human Behavior, 23, 2904-2927. doi:10.1016/j.chb.2006.06.005

Bandura, A. (1993). Perceived Self-Efficacy in Cognitive Development and Functioning. Educational Psychologist. doi:10.1207/s15326985ep2802_3

Bandura, A. (2012). On the Functional Properties of Perceived Self-Efficacy Revisited. Journal of Management. doi:10.1177/0149206311410606

Barnes, S. J., \& Pressey, A. D. (2014). Caught in the Web? Addictive behavior in cyberspace and the role of goal-orientation. Technological Forecasting and Social Change, 86, 93-109. doi:10.1016/j. techfore.2013.08.024

Baumann, M. (1998). Time pressure, performance and awareness of group members in co-acting groups. In AMCIS Proceedings (pp. 240-242). Retrieved from http://www.comp.nus.edu.sg/ cs6380/ AMCIS_Baumann.pdf

Bock, G. W., Ko, D. G., Kirsch, L. J., \& King, W. R. R. (2005). Behavioral Intention Formation in Knowledge Sharing: Examining the Roles of Entrinsic Motivators, Social- Phychological Forces and Organizational Climate. JSTOR: MIS Quarterly, Vol. 29, 87-111. Retrieved from http://www.jstor.org/ 
discover $/ 10.2307 / 25148668$ ? uid=3738672\&uid=2\&uid=4\&sid=21103966307491 \nhttp://www.jstor. org $/$ discover $/ 10.2307 / 25148669$ ?uid=3738672\&uid=2\&uid=4\&sid=21103966307491

Brown, S. A., Dennis, A. R., Burley, D., \& Arling, P. (2013). Knowledge Sharing and Knowledge Management System Avoidance : The Role of Knowledge Type and the Social Network in Bypassing an Organizational Knowledge Management System. Journal of the American Society for Information Science and Technology, 64(July), 2013-2023. doi:10.1002/asi

Csikszentmihalyi, M. (1990). Flow: The psychology of optimal experience. Optimal experience: Psychological studies of flow in consciousness (1st ed., p. 336). Harper Perennial Modern Classics.

Csikszentmihalyi, M., Abuhamdeh, S., \& Nakamura, J. (2005). Flow. In Handbook of competence and motivation. (pp. 598-608).

DeSmet, A., Van Ryckeghem, D., Compernolle, S., Baranowski, T., Thompson, D., Crombez, G., ... De Bourdeaudhuij, I. (2014). A meta-analysis of serious digital games for healthy lifestyle promotion. Preventive Medicine, 69, 95-107. doi:10.1016/j.ypmed.2014.08.026

Deterding, S., Dixon, D., Khaled, R., \& Nacke, L. (2011). From game design elements to gamefulness. In Proceedings of the 15th International Academic MindTrek Conference on Envisioning Future Media Environments - MindTrek'11 (p. 9). New York, New York, USA: ACM Press. doi:10.1145/2181037.2181040

Hamari, J. (2013). Transforming homo economicus into homo ludens: A field experiment on gamification in a utilitarian peer-to-peer trading service. Electronic Commerce Research and Applications, 12(4), 236-245. doi:10.1016/j.elerap.2013.01.004

Hamari, J., \& Koivisto, J. (2014). Measuring flow in gamification: Dispositional Flow Scale-2. Computers in Human Behavior. doi:10.1016/j.chb.2014.07.048

Hamari, J., Koivisto, J., \& Sarsa, H. (2014). Does Gamification Work? -- A Literature Review of Empirical Studies on Gamification. In 2014 47th Hawaii International Conference on System Sciences (pp. 3025-3034). IEEE. doi:10.1109/HICSS.2014.377

Hansen, M. T., \& von Oetinger, B. (2001). Introducing T-shaped managers. Knowledge management's next generation. Harvard Business Review, 79, 106-116, 165. doi:10.1108/00483488910133459

Hartmann, T., \& Klimmt, C. (2006). Gender and computer games: Exploring females' dislikes. Journal of Computer-Mediated Communication, 11, 910-931. doi:10.1111/j.1083-6101.2006.00301.x

Hasan, B. (2006). Effectiveness of computer training: The role of multilevel computer self-efficacy. Journal of Organizational and End User Computing, 18, 50-67. Retrieved from http://www.scopus. com/inward/record.url?eid=2-s2.0-33645563213\&partnerID=tZOtx3y1

He, W., \& Wei, K. K. (2009). What drives continued knowledge sharing? An investigation of knowledge-contribution and -seeking beliefs. Decision Support Systems, 46, 826-838. doi:10.1016/j. dss.2008.11.007

Heaven, D. (2014). Engineered compulsion: why Candy Crush is the future of more than games. New Scientist, 222(2971), 38-41. doi:10.1016/S0262-4079(14)61069-1

Hofstede, G., \& Hofstede, G. J. (2006). Kultury a organizace: Software lidské mysli (p. 335). Praha: Linde.

Kilduff, G. J. (2014). Driven to Win: Rivalry, Motivation, and Performance. Social Psychological and Personality Science, 5(8), 944-952. doi:10.1177/1948550614539770

Koivisto, J., \& Hamari, J. (2014). Demographic differences in perceived benefits from gamification. Computers in Human Behavior, 35, 179-188. doi:10.1016/j.chb.2014.03.007

Kuo, R.-Z., \& Lee, G.-G. (2011). Knowledge management system adoption: exploring the effects of empowering leadership, task-technology fit and compatibility. Behaviour \& Information Technology. doi:10.1080/0144929X.2010.516018

Lai, J. Y. (2009). How reward, computer self-efficacy, and perceived power security affect knowledge management systems success: An empirical investigation in high-tech companies. Journal of the American Society for Information Science and Technology, 60, 332-347. doi:10.1002/asi.20982

Liebeskind, J. P. (1996). Knowledge, Strategy, and the Theory of the Firm. Strategic Management Journal, 17, 93-107. doi:10.2307/2486993 
Maslow, A. H. (1943). A theory of human motivation. Psychological Review. doi:10.1037/h0054346

McCrae, R. R., \& Costa, P. T. (1989). The structure of interpersonal traits: Wiggins's circumplex and the five-factor model. Journal of Personality and Social Psychology, 56, 586-595. doi:10.1037/00223514.56.4.586

Meder, M., Plumbaum, T., Luca, E. W. De, \& Albayrak, S. (2011). Gamification : A Semantic Approach for User Driven Knowledge Conservation. In FGWM 2011 -Workshop on Knowledge and Experience Management at LWA 2011 (pp. 15-18).

Moore, D. A., \& Tenney, E. R. (2012). Time Pressure, Performance, and Productivity. In Looking Back, Moving Forward: A Review of Group and Team-Based Research (Research on Managing Groups and Teams, Volume 15) (pp. 305-326). Emerald Group Publishing Limited. Retrieved from http://www. emeraldinsight.com/doi/abs/10.1108/S1534-0856(2012)0000015015

Morris, M. G., \& Venkatesh, V. (2000). Age Differences in Technology Adoption Decisions: Implications for A Changin Work Force. Personnel Psychology, 53, 375-403. doi:10.1111/j.1744-6570.2000.tb00206.x

Morris, M. G., Venkatesh, V., \& Ackerman, P. L. (2005). Gender and age differences in employee decisions about new technology: an extension to the theory of planned behavior. IEEE Transactions on Engineering Management, 52. doi:10.1109/TEM.2004.839967

Pedreira, O., García, F., Brisaboa, N., \& Piattini, M. (2014). Gamification in software engineering - A systematic mapping. Information and Software Technology, 57, 157-168. doi:10.1016/j. infsof.2014.08.007

Reeves, B., \& Read, J. L. (2009). Total Engagement: Using Games and Virtual Worlds to Change the Way People Work and Businesses Compet. Harvard Business School Press Books, 1. doi:10.1016/j. chb.2010.03.035

Rinc, S. (2014). INTEGRATING GAMIFICATION WI TH KNOWLEDGE MANAGEMENT. In Management, Knowledge and Learning International Conference (pp. 997-1003). Portoroz.

Schein, E. H. (2010). Organizational culture and leadership. Leadership (4th ed., Vol. 2, p. 464). San Francisco: Jossey-Bass. doi:10.1016/j.sbspro.2011.12.156

Trees, L. (2013). Gamification in Knowledge Management. APQC Overview. Retrieved from http:// www.apqc.org/knowledge-base/documents/gamification-knowledge-management-apqc-overview

Venkatesh, V., Morris, M. G., Davis, G. B., \& Davis, F. D. (2003). User acceptance of information technology: Toward a unified view. Management Information Systems Quarterly, 27, 425-478. doi: $10.2307 / 30036540$

Wang, S., Noe, R. A., \& Wang, Z.-M. (2011). Motivating Knowledge Sharing in Knowledge Management Systems: A Quasi-Field Experiment. Journal of Management, 0149206311412192-. doi:10.1177/0149206311412192

Wang, W.-T., \& Lai, Y.-J. (2014). Examining the adoption of KMS in organizations from an integrated perspective of technology, individual, and organization. Computers in Human Behavior, 38, 55-67. doi:10.1016/j.chb.2014.05.013

Wasko, M. M., \& Faraj, S. (2005). Why should I share? Examining social capital and knowledge contribution in electronic networks of practice. MIS Quarterly, 29, 35-57. doi:Article

Williams, D., Consalvo, M., Caplan, S., \& Yee, N. (2009). Looking for gender: Gender roles and behaviors among online gamers. Journal of Communication, 59, 700-725. doi:10.1111/j.14602466.2009.01453.x

Wolfinger, P. (2013). Game On: How Gamification Is Changing Business. Society for Human Resource Management. Retrieved from http://www.shrm.org/hrdisciplines/technology/articles/pages/playinggames-at-work.aspx

Michal Ďuriník durinik.michal@gmail.com

Masaryk University Brno, Faculty of Economics and Administration 